\title{
MODAL THEORY OF SKIN EFFECT IN SINGLE AND MULTIPLE TURN COILS
}

\author{
P. Silvester \\ McGill University \\ Montreal, 110, P. Q. \\ Canada
}

\author{
S. K. Wong \\ California Institute \\ of Technology \\ Pasadena, California
}

\author{
P. E. Burke \\ University of Toronto \\ Toronto, Ontario \\ Canada
}

\begin{abstract}
The skin effect in circularly symmetric structures is formulated in terms of an integral equation, which may be solved in terms of the normal modes of the corresponding integral equation eigenvalue problem. The necessary modal functions are computed numerically, using a simple discrete model of the coil. The modal series solution easily accounts for electrical excitation of the coil as for external magnetic fields. A comparison with so-called "direct" calculation methods, as well as with published experimental results, shows that the method is capable of good accuracy, while effecting substantial economies in computation.
\end{abstract}

\section{INTRODUCTION}

The problem of predicting current distribution in single-turn and multi-turn coils has recently arisen in connection with induction heating problems, and methods for its solution have been proposed in several papers $[1,2]$. Nearly all such methods have relied on modelling the coil turn or turns in question by a set of parallel-connected filaments, whose self and mutual impedances are evaluated so as to permit the determination of current distribution in the sinusoidally time-varying case. These techniques have exhibited several disadvantages. First, numerical instabilities appear to have plagued attempts at solution, and it has in some cases been necessary to introduce deliberate distortions in the physical model in order to regain numerical stability [3]. Secondly, the matrix of impedances that describes any one filament model is only valid at one frequency, and solution of a geometrically similar problem with different frequency, or resistivity, requires setting up the entire problem anew, without in any way using the results obtained previously. Third, the restriction to a single-frequency operation makes it quite difficult to evaluate the effects of nonsinusoidal waveforms, and entirely precludes solving the problem of transient skin effect. Similar difficulties are encountered in attempts to solve the skin-effect problem in straight, infinitely long conductors [4]. These complications, however, have been shown to be largely avoidable in the straight-conductor case by solving in terms of modal functions $[5,6,7]$, instead of filament currents.

The object of the present paper is to show that the modal theory of skin effect can be used to solve rotationally symmetric problems with no more difficulty than the long, straight conductor case. External magnetic fields as well as directly imposed electrical excitations are readily accounted for in the solutions, so that the method serves just as well for multi-turn coils as for calculating the eddy currents in a single short-circuited turn. In fact, there is no need to model any but a few turns in detail, so that no restriction need be placed on the number of coil turns for which solution is to be carried out. Digital computer programs to carry out such solutions have been written, and are described below.

\section{CURRENT DISTRIBUTION IN A COIL}

Let a coil of one or more turns be considered, whose axis coincides with the $\mathrm{z}$ axis of a cylindrical coordinate system. If the spiralling

Paper 71 TP 523-PWR, recommended and approved by the Rotating Machinery Committee of the IEEE Power Engineering Society for presentation at the IEEE Summer Meeting and International Symposium on High Power Testing, Portland, Ore., July 18-23, 1971. Manuscript submitted June 22, 1970; made available for printing April 27, 1971. of the turns is neglected, each turn may be thought to constitute a ring, so that the coil is approximated by a set of concentrically placed rings. Throughout each ring, the current density $\mathrm{J}$, electric field $\mathrm{E}$, and the magnetic vector potential $\mathrm{A}$ will be assumed to possess only azimuthal components; their axial and radial components will be taken to be zero.

At any point $P$ in an energized coil, the magnetic vector potential is

$$
A_{P}=\int_{S} G(P ; Q) J_{Q} d S_{Q}+A_{e}
$$

where $J_{Q}$ is the current density at another point $Q$ in the same coil, $G(P ; Q)$ is the appropriate Green's function $[8,9]$, and $A_{e}$ is the magnetic vector potential due to currents external to the ring. The integration is over the whole turn cross-section denoted by $\mathrm{S}$.

The applied electric field must always equal the sum of the ohmic drop and the back electromotive force. That is,

$$
E_{p}=\frac{J_{p}}{g_{p}}+\frac{\partial A_{p}}{\partial t}
$$

where gP is the local conductivity, Combining (1) and (2), and rearranging, there is obtained

$$
J_{P}+\int_{S} G(P ; Q) g_{P} \frac{\partial J_{Q}}{\partial t} d S_{Q}=g_{P}\left(E_{P}-\left.\frac{\partial A}{\partial t}\right|_{P}\right)
$$

Since all problem boundaries are time-invariant, the classical process of setting up the current density in the form of a product of space and time components [7], say $J=T(t) J(x, y)$ may be used. The homogeneous equation associated with (3) then becomes

$$
J_{P}+\lambda \int_{S} G(P ; Q) g_{P} J_{Q} d S_{Q}=0
$$

where $\lambda$ is a separation constant, and the current densities refer to space-variant components only.

Equation (4) is the conventional eigenvalue problem of a Fredholm integral equation. The only inconvenience is that the kerriel function $G(P ; Q) g_{P}$ is not symmetric in the points $P$ and $Q$. However, it may be symmetrized readily; it is only necessary to multiply it by $\sqrt{r_{\mathrm{p}} / \mathrm{g}_{\mathrm{P}}}$, where $\mathrm{r}$ is the radial position of the point $\mathbf{P}$. Then, defining a weighted current density $\psi$, and a symmetrized kernel $\mathrm{K}(\mathrm{P} ; \mathrm{Q})$, by

$$
\begin{aligned}
& \psi=J \sqrt{r / g} \\
& K(P ; Q)=\sqrt{g_{P} g_{Q}} G(P ; Q) \sqrt{r_{P} / r_{Q}}
\end{aligned}
$$

equation (4) assumes the perfectly symmetric form

$$
\psi(P)-\frac{1}{\lambda} \int_{S} K(P ; Q) \psi(Q) d S_{Q}=0
$$

This symmetry guarantees that all the eigenvalues $\lambda$ will be real. Let $\lambda_{i}$ and $\psi_{i}, i=1,2,3, \ldots$, denote the eigenvalues and corresponding eigenfunctions which satisfy (7). 
To solve the inhomogeneous problem defined by equation (3), let steady-state sinusoidal excitations be considered. Then (3) may be written

$$
J_{P}+j \omega \int_{S} G(P ; Q) g_{P} J_{Q} d S_{Q}=g_{P}\left(E-j \omega A_{e}\right)_{P}
$$

Again multiplying by $\sqrt{r_{\mathrm{P}} / \mathrm{g}_{\mathrm{P}}}$, there obtains

$$
\begin{aligned}
\sqrt{\frac{r_{P}}{g_{P}}} J_{P}+j \omega \int_{S} G(P ; Q) \frac{\sqrt{g_{P} g_{Q}}}{\sqrt{r_{Q} r_{P}}} \sqrt{\frac{r_{Q}}{g_{Q}}} J_{Q} d S_{Q} \\
=\sqrt{g_{P}}\left(E-j \omega A_{e}\right)_{P} \sqrt{r_{P}}
\end{aligned}
$$

Expanding in series of eigenfunctions, set

$$
\sqrt{\frac{r}{g}} J=\sum_{i=1}^{\infty} c_{i} \psi_{i}
$$

and

$$
\sqrt{g r}\left(E-j \omega A_{e}\right)=\sum_{i=1}^{\infty} b_{i} \psi_{i}
$$

Substitute the above expansion in eqn. (9). There results

$$
\sum_{i=1}^{\infty} c_{i} \psi_{i}+i \omega \sum_{i=1}^{\infty} c_{i} \int_{S} K(P ; Q) \psi_{Q i} d S Q=\sum_{i=1}^{\infty} b_{i} \psi_{i}
$$

In view of equation (7), the integral in (12) is easily evaluated and there is obtained

$$
\sum_{i=1}^{\infty} c_{i} \psi_{i}+\sum_{i=1}^{\infty} j \omega c_{i} \lambda_{i} \psi_{i}=\sum_{i=1}^{\infty} b_{i} \psi_{i}
$$

Rearranging (13),

$$
\sum_{i=1}^{\infty}\left[\left(1+j \omega \lambda_{i}\right) c_{i}-b_{i}\right] \psi_{i}=0
$$

Since the eigenfunctions $\psi_{\mathrm{i}}$ are not only linearly independent but mutually orthogonal, equation (14) must have equality term by term:

$$
c_{i}=\frac{b_{i}}{1+j \omega \lambda_{i}} \quad, \text { for } i=1,2,3, \ldots
$$

That is, given the $b_{i}$, the $c_{i}$ can be evaluated. Since $E$ and $A_{e}$ are given, expansion (11) is always possible. Subsequently, equation (10) will give the prescription for synthesizing the current distribution $J$ out of

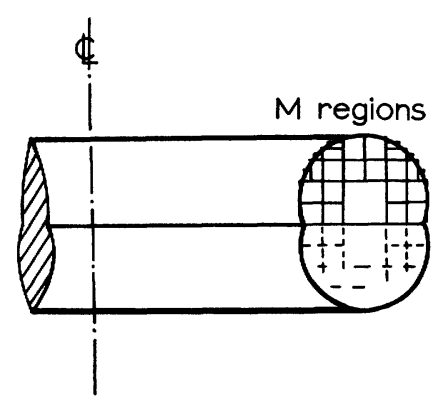

Fig. 1. Subdivision of axisymmetric ring or coil turn into sub-rings or regions. Note that all regions need not be of the same size, and that use may be made of symmetry. the $c_{i}$ and $\psi_{i}$, thereby solving the problem provided the eigenvalue problem (7) can be solved so as to obtain the necessary $\lambda_{i}$ and $\psi_{i}$.

\section{COMPUTATION OF THE EIGENFUNCTIONS}

The final solution stage, equations (8) to (15), can be carried out without difficulty, provided (a) a way is known for finding the eigenvalues and eigenfunctions, (b) the infinite series expansions can be truncated at some convenient number of terms with acceptable error, and (c) the expansion coefficients in (10) and (11) can be determined. To determine approximations to the eigenvalues and eigenfunctions, the region of integration is subdivided into smaller regions $S_{k}$, with $\mathrm{k}=1,2,3, \ldots \mathrm{M}$, as in Figure 1. Averaging $\psi$ in the exact integral equation (7) over the $\mathrm{k}^{\text {th }}$ subdivision yields

$$
\Psi_{k}+\sum_{i=1}^{M} \lambda \frac{1}{S_{k}} \int_{S_{k}} \int_{S_{i}} K(k ; i) d S_{i} d S_{k} \Psi_{i}=0
$$

in which $\Psi$ represents the average value of $\psi$ over the $\mathrm{k}^{\text {th }}$ subdivision. Symmetrization of (16) is achieved by multiplication by $\sqrt{\mathrm{S}_{\mathrm{k}}}$ :

$$
\sqrt{S_{k}} \Psi_{k}+\sum_{i=1}^{M} \frac{\lambda}{\sqrt{S_{k} S_{i}}} \sum_{S_{k}}^{j} \int_{S_{i}} K(k ; i) d S_{i} d S_{k} \sqrt{S_{i}} \Psi_{i}=0
$$

Now, define a matrix $K$ and a vector $\Phi$ :

$$
\begin{aligned}
& K_{i k}=\frac{1}{\sqrt{S_{k} S_{i}}} \int_{S_{k}} \int_{i} K(k ; i) d S_{j} d S_{k} \\
& \Phi=S_{k} \Psi_{k}
\end{aligned}
$$

Then (17) becomes a matrix equation of order M:

$$
(U+\lambda K) \Phi=0
$$

which is an eigenvalue problem in almost exactly standard form, $\mathrm{U}$ representing the unit matrix. Library routines are readily available for the solution of (20). It is instructive to examine the $\mathrm{K}$ matrix in detail. By its definition,

$$
\begin{gathered}
K_{i k}=\frac{\sqrt{g_{k} S_{k}} \sqrt{g_{i} S_{i}}}{2 \pi \sqrt{r_{k}{ }^{r}}} \frac{1}{S_{k} S_{i}} \\
\int_{S_{k} S_{i}} 2 \pi \sqrt{r_{k}{ }^{r}} G(k ; i) \sqrt{\frac{r_{k}}{r_{i}}} d S_{i} d S_{k}
\end{gathered}
$$

If a diagonal matrix $\mathrm{R}$ and a matrix $\mathrm{L}$ are defined by

$$
\begin{aligned}
& R_{k k}=\frac{2 \pi r_{k}}{g_{k} S_{k}} \\
& L_{i k}=\frac{1}{S_{i} S_{k}} \int_{\xi_{k}} \int_{i} 2 \pi r_{k} G(k ; i) d S_{i} d S_{k}
\end{aligned}
$$

then on substitution, (21) becomes

$$
K=\operatorname{DIAG} \cdot\left\{\frac{1}{\sqrt{\mathrm{R}_{k k}}}\right\} \quad \mathrm{D} \quad \operatorname{DIAG} \cdot\left\{\frac{1}{\sqrt{\mathrm{R}_{k k}}}\right\}
$$


However, (22) and (23) exactly define the matrices of resistances and inductances (in the conventional sense) of the circular subsections of area $S_{k}$ into which the typical coil turn has been divided. Thus, starting from a purely field-theoretic viewpoint, a discrete representation has been obtained which coincides with the simple filament models used in earlier work. The similarity with the corresponding formulations obtained for straight conductors is at once evident.

In order to avoid cluttering programs with repeated dimensional information, the matrix $\mathrm{K}$ is replaced by a normalized matrix $\mathrm{K}$,

$$
\bar{K}=\frac{1}{\mu_{0} g r_{0}^{2}} K
$$

$\overline{\mathrm{K}}=\mathrm{K}$ is obtained for unity absolute conductivity and permeability, and unity reference radius $r_{0}$. The matrix $\bar{K}$ has normalized eigenvalues $\bar{\lambda}$ which are related to the true $\lambda$ obviously by:

$$
\lambda=\frac{1}{\mu_{0} g r_{0}^{2}} \bar{\lambda}
$$

Hence, the eigenvalue problem to be solved is

$$
(U+\dot{\bar{\lambda}} \bar{K}) \emptyset=0
$$

It will be assumed that all eigenvectors are normalized to the same length by the available eigenvalue problem subroutines.

\section{SOLUTION OF THE INHOMOGENEOUS PROBLEM}

The calculated eigenvalues and eigenvectors may now be used to solve the inhomogeneous equation (8) in exactly the manner indicated in equations (10) - (15). For computation, however, it is convenient to replace the latter, which are continuum equations, by corresponding matrix equations. For this purpose, let the vectors $V$ and $\Phi$ be defined by

$$
\begin{aligned}
& V_{i}=\left(E-j \omega A_{e}\right)_{i} 2 \pi r_{i} /\left(g_{i} S_{i}\right) \\
& \Phi_{i}=J_{i} S_{i} \sqrt{R_{i i} / 2 \pi}
\end{aligned}
$$

and the normalized frequency $\Omega$, similarly to the straight-conductor case, by

$$
\Omega=\omega \mu_{0} g r_{0}^{2}
$$

With these definitions, an averaging process exactly like that described above for equation (16), converts equation (9) into the simple matrix equation

$$
(U+i \Omega \bar{K}) \Phi \sqrt{2 \pi}=R^{-1} V
$$

The expansion corresponding to equation (11) may be accomplished by setting

$$
R^{-1} V=\sum_{k=1}^{M} b_{k} q_{k}
$$

where $\Phi_{k}$ denotes the $\mathrm{k}^{\text {th }}$ eigenvector of equation (20), corresponding to the eigenvalue $\bar{\lambda}_{k}$, and the coefficients $b_{i}$ are as yet undetermined. To determine them premultiply both sides by $\Phi^{T}$, the transposed $i^{\text {th }}$ eigenvector. Because the eigenvectors are orthonormal (a consequence of the symmetry of $\overline{\mathrm{K}}$ ), zeros result except for $\mathrm{j}=\mathrm{k}$ :

$$
\Phi_{i}^{\top} R^{-1} V=\begin{aligned}
& 0, i \neq k \\
& b_{k}, i=k
\end{aligned}
$$

Hence the desired coefficients $c_{k}$, equation (15), are approximated by

$$
c_{k}=\frac{\phi_{k}^{\top} R^{-1} V}{1+i \bar{\lambda}_{k}}
$$

Since both the coefficients, and approximate eigenfunctions, are now in hand, synthesis of the current distributions themselves is a fairly straightforward matter.

It will be noted that the summation in equation (32) need not be taken to cover all the eigenfunctions that can be obtained from solving equation (27), but only some restricted number $M$. Since the eigenfunctions are all mutually orthogonal, it is known that the series (32) is uniformly convergent; and practice shows $15-20$ terms to be more than ample for good convergence to be achieved. In other words, regardless of the fineness of subaivision used to model the conductor cross-section, the number of terms used in (32) remains around 15-20 or even fewer. It is this fact which is largely responsible for the substantial computational economies achievable by the modal method; the "direct" solution schemes [1,2,4] involve matrix operations of order 100-200, which are much more time-consuming.

\section{SOLUTION FOR PRESCRIBED TOTAL CURRENT}

The above discussion presupposes that the forcing function E$\mathrm{j} \omega \mathrm{A}_{\mathrm{e}}$ is known. More commonly, however, the total current in the coil is prescribed, and the value of $\mathrm{E}$ must indeed be determined as part of the problem itself. In this case, a solution may be sought as follows. First, take $\mathrm{E}=0$; this assumption will produce the part of current density caused by the induced emf due to $A_{e}$. The total current density, by superposition, is composed of two terms, a certain $\mathrm{J}_{\mathrm{E}}$ associated with the applied electric field $\mathrm{E}$ and the other, say $\mathrm{J}_{\mathrm{A}}$, associated with the magnetic vector $A_{e}$ :

$$
J=J_{E}+J_{A}
$$

where

$$
J_{E P}+j \omega \int_{S} G(P ; Q) g J_{E Q} d S_{Q}=g E_{P}
$$

and

$$
J_{A P}+j \omega \int_{S} G(P ; Q) g J_{A Q} d S_{Q}=j \omega g A_{e}(P)
$$

Since $A_{e}$ is known, finding $J_{A}$ is straight forward, using the method detailed above. Next, let $\mathrm{E}$ have a prescribed uniform value, say $\mathrm{E}=1$, and solve equation (36) to find the corresponding $\mathrm{J}_{\mathrm{E}}$. For any other $\mathrm{E}=\mathrm{V}$, the corresponding current density will be $\mathrm{VJ}_{\mathrm{E}}$. To meet the requirement that the turn current must be equal to the given coil current, say $I_{t}$, the total current may be expressed as

$$
I_{t}=V \int_{S} J_{E} d S+\int_{S} J_{A} d S
$$

The only unknown in this equation is $\mathrm{V}$, which may now be solved for:

$$
V=\frac{I_{t}-\int_{A} J_{A} d S}{\int_{S} J_{E} d S}
$$


If the necessary applied voltage for the whole coil, or any related quantity, is desired, the above algorithm may be applied to all the turns in the coil, one at a time. If, as is quite common, the cross-sectional shape of all turns is the same, the modal functions and eigenvalues will also be the same; regardless of the number of turns in the coil, "stepping" through all the turns only requires recalculation of $A_{e}$ and $\mathrm{J}_{\mathrm{A}}$ for each turn. Since this implies only a summation of 15-20 terms without any new matrix operations, the new method clearly has a particularly great advantage in applications of this type.

\section{COMPUTER PROGRAM CHARACTERISTICS}

A set of computer programs, embodying the methods described above, has been written. The crucial pair of programs, obviously, consists of one for setting up and solving the eigenvalue problem (so as to determine the modal functions and normalized eigenvalues), and another for combining together the modal solutions in series so as to find actual current distributions. There is no need for these two programs to have anything in common. Indeed, there is good reason to keep them entirely separate, in order to save memory space and so as to permit solutions for various material parameters, turns, numbers, frequencies, etc. without wasting time in needless recomputation of the modal functions. A third program, wholly optional, has also been written to perform subdivision of large turns into filaments semiautomatically, so that for reasonably regular cross-sectional shapes, very little input data need be prepared. This program is useful in eliminating the almost inevitable human error involved in preparing substantial quantities of numerical data.

A basic question of interest relates to the speed and cost of running these programs. As already indicated above, the synthesis of current distribution from the modal functions for any given normalized frequency is very fast, verging on the trivial where cost is concerned; at most, the computational expense is measured in cents per solution. Indeed, the majority of the computing cost in most solutions lies in what might be termed data handling operations, e.g., conversion of complex numbers from rectangular to polar form, calculation of root-mean-square values, and printing out answers. The eigenvalue problem, on the other hand, is more costly, and it is here that comparison with so-called "direct" methods $[1,4]$ is necessary. In the latter methods, each new problem requires the solution of a system of $\mathrm{N}$ complex linear algebraic equations, where $\mathrm{N}$ is the number of filamentary subconductors. The corresponding modal treatment requires solution of an eigenvalue problem of order N. If Gaussian triangular decomposition (the fastest general method) is used, the former involves somewhat more than $\mathrm{N}^{3} / 3$ complex arithmetic multiply-andadd operations (equivalent to roughly $\mathrm{N}^{3}$ real operations, though the precise conversion factor is machine dependent). Using Householder tridiagonalization, followed by Sturm sequences and inverse iteration (the fastest general method for the symmetric eigenproblem), somewhat over $2 \mathrm{~N}^{3} / 3$ real operations are needed. The initial overhead in setting up the matrices is very nearly equal in either case. One may conclude, therefore, that for one single solution, either method may be slightly faster, depending on whether the available computer relies on software or hardware implementation of complex arithmetic. For subsequent solutions, the computational cost is very small if modal series are employed. Where core memory capacity is a limitation, the modal method is to be recommended in any case, since a real matrix requires exactly half as much memory space as a complex matrix of the same order.

It should further be emphasized that, because of the orthonormality of modal functions, numerical stability is absolutely guaranteed for the individual solutions. The eigenvalue problem itself, of course, is not sensitive to ill conditioning of the matrices; even if the matrix $\overline{\mathrm{K}}$ should have one of its eigenvalues exactly zero, all eigenvalues and eigenfunctions will still be computed without any difficulty. Triangular decomposition, on the other hand, would fail altogether in the latter case.
To summarize, the modal programs are, at best, much faster than those based on filamentary current analysis; at their worst, their performance equals that of the latter. In terms of required memory capacity, they are invariably better by a factor of two, resulting from the advantage of real over complex arithmetic. They do not suffer from the numerical instabilities which have been reported by other investigators using techniques sensitive to ill conditioning of matrices. These theoretically evident advantages have been extensively verified by computing experience.

\section{COMPARISON WITH EXPERIMENTAL RESULTS}

In order to verify the operation of the programs described above, and to provide information on the effects of various degrees of fineness of modelling, truncation of the infinite modal series, and other computational variables, computed results were compared with three sets of experimental data. Measurements have been made on mercury coils, using current density measuring probes [9], and computations have been carried out both by the modal series method and a direct filament-current modelling technique.

The first experiment concerns a single-turn mercury coil of square section, Figure 2, fed with $400 \mathrm{~Hz}$. current. In this case, $A_{e}=0$ in equation (1), and only the directly applied electric field $\mathrm{E}$ need be considered. Results obtained in this case are shown in Figure 3; it is seen that agreement is good.

The second experiment concerns a short-circuited single-turn coil or annular billet, Figure 4, concentrically within a solenoid. The solenoid is fed with current at $10 \mathrm{kHz}$, and current densities in the short-circuited turn are measured. For purposes of calculation, there is in this case no impressed $\mathrm{E}$, and the excitation consists of an externally supplied vector potential $A_{e}$ (due to the solenoid turns) only. As may be seen from Figure 5, the results are again in good agreement.

In both Figures 3 and 5, it may be noted on careful examination that the current density distribution computed by modal series exhibits one of the usual characteristics of least-square solutions: it oscillates slightly above and below the true values, so as to be slightly

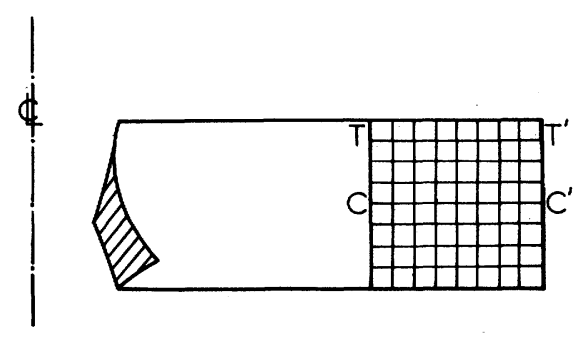

Fig. 2. Modelling of single-turn mercury coil. The outer radius of the coil is $15 \mathrm{~cm}$., the frequency of excitation, $400 \mathrm{~Hz}$.

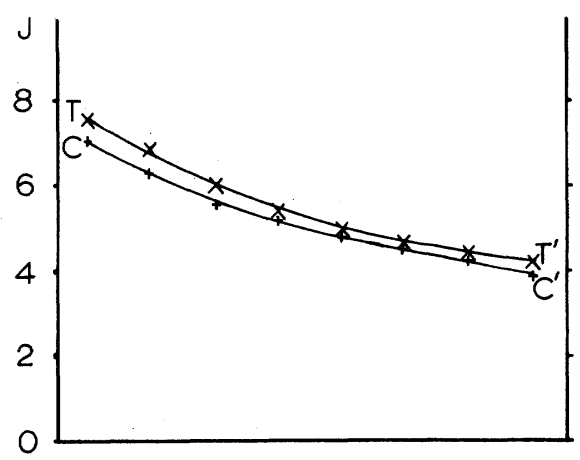

Fig. 3. Measured and predicted current densities in the mercury coil turn, along the top $\left(T-T^{\prime}\right)$ and centre $\left(C-C^{\prime}\right)$ planes. The solid line denotes predicted, crosses experimental, values. 


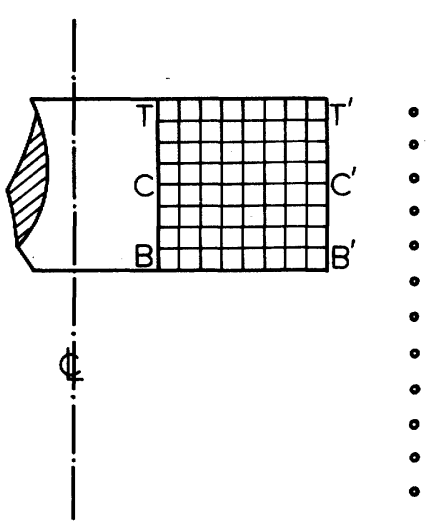

Fig. 4. Subdivision of a short-circuited mercury coil, of $7.5 \mathrm{~cm}$. outer radius, placed within a solenoidal coil located in the position indicated by circles. Excitation frequency in this case is $10 \mathrm{kHz}$.

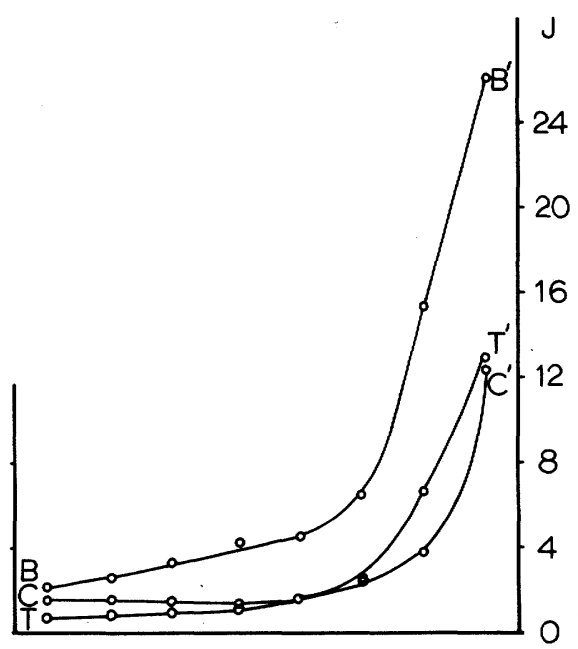

Fig. 5. Measured and predicted current densities along the top (T-T'), centre $\left(C-C^{\prime}\right)$ and bottom $\left(B-B^{\prime}\right)$ planes of the annular billet of Figure 4. Solid lines denote predicted, circles experimental values.

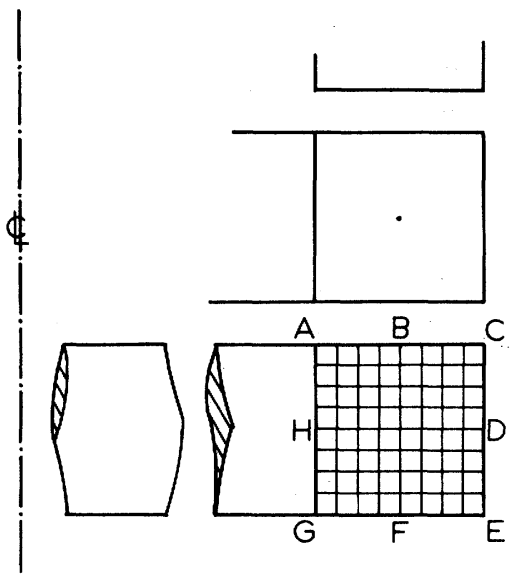

Fig. 6. Modelling of the end turn of a 15-turn mercury coil. The coil length is $23.5 \mathrm{~cm}$., outer radius $22.9 \mathrm{~cm}$., and conductor cross section $12.7 \mathrm{~mm}$. square. The coil is excited with 1 ampere at a frequency of $10 \mathrm{kHz}$.

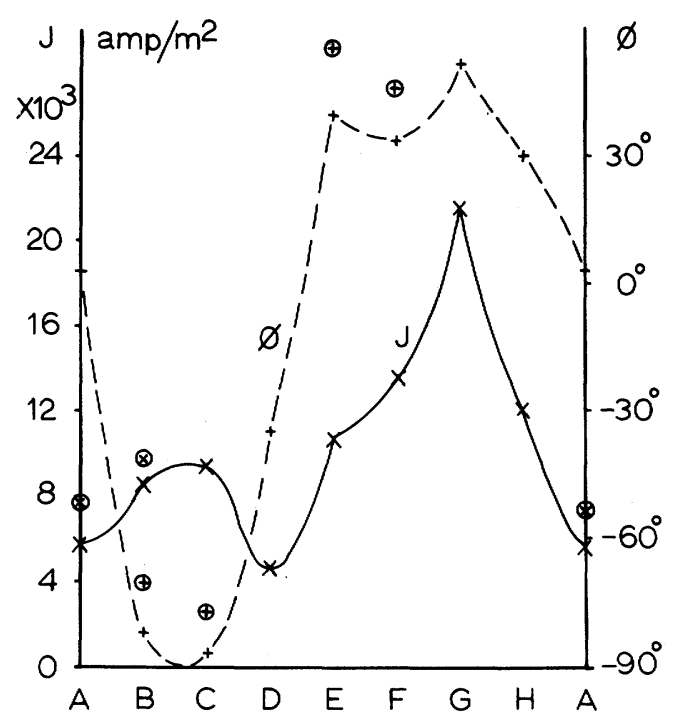

Fig. 7. Predicted and measured current densities in the end turn of Figure 6, along the conductor periphery (measurements made in positions denoted by letter symbols). The solid and dashed lines represent values predicted by modal theory; crosses denote predicted values given by filament (coupled-circuit) methods; circles are measured points.

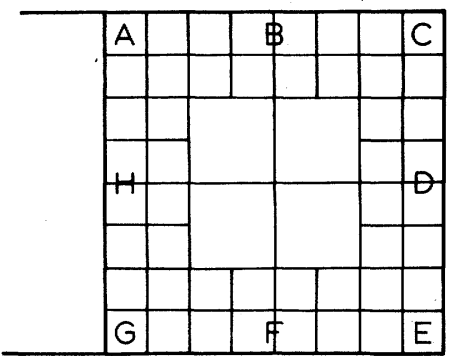

Fig. 8. An alternative subdivision of the coil turn of Figure 6. The smaller number of subdivisions results in shorter computing times, while accuracy is essentially unaffected.

in error almost everywhere, and badly wrong nowhere. This behaviour is well known from Fourier series, which are perhaps the orthogonal series expansions most familiar to many electrical engineers.

Figure 6 shows the subdivision for calculating current density distribution in the end turn of a 15-turn mercury coil excited by a prescribed current at $10 \mathrm{kHz}$. In this case, only the end turn is examined, so that both the impressed field $\mathrm{E}$ and the vector potential $A_{e}$ caused by the other fourteen turns must be taken into account. The modal solution and filamentary model solution match very well, Figure 7; there is a slight deviation from experimental values, probably caused by the fact that the measured results refer to the outside surface of the turn, while the computed values represent averages over a finite thickness of conductor material. Nevertheless, the agreement is held to substantiate the method of calculation.

Another, coarser, subdivision of the same coil turn is shown in Figure 8. Recalculation with this subdivision yields results within $1 \%$ of those in Figure 7, showing that a fine subdivision in the interior of the conductor may be dispensed with, with attendant savings in computer time.

\section{CONCLUSIONS}

It has been found possible to reformulate the modal theory of skin effect so as to apply to circularly symmetric geometries as well as to long, straight conductors. Although the theory is slightly more 
complicated, its application in this case does not require significant extra programming effort, nor increased computing time or storage. In solving problems of current distribution in coils, where solution becomes possible one turn at a time, the modal method holds significant advantages and promises to allow treatment of a variety of practical problems at the expenditure of relatively little computing time. There appears every reason to believe that this method is the most economically, as well as the most stable numerically, of those developed to date.

\section{REFERENCES}

[1] P. F. Ryff, P. P. Biringer, P. E. Burke, "Calculation methods for current distribution in single-turn coils of arbitrary cross section," IEEE TRANS. POWER APPARATUS AND SYSTEMS, vol. 89, pp. 228-232, February 1970.

[2] P. E. Burke, P. P. Biringer, P. F. Ryff, E. Solger, "The prediction and measurement of current distribution in coaxial circular geometries," IEEE PICA Conference, Denver, 1969.

[3] P. P. Biringer, P. F. Ryff, R. S. Segsworth, "Current distribution in conductors of arbitrary cross section considering corner effects," Conference Record 1968 IEEE IGA Group Ann. Meeting (Chicago, Ill.), pp. 105-116.

[4] G. Schaffer, P. Banderet, "Skin effect in heavy-current conductor bars," Brown Boveri Rev., vol. 52, pp. 623-628, August 1965.

[5] P. Silvester, "Modal network theory of skin effect in flat conductors," Proc. IEEE, vol. 54, pp. 1147-1151, September 1966.

[6] P. Silvester, "The accurate solution of skin effect in conductors of complicated shape," IEEE TRANS. POWER APPARATUS AND SYSTEMS, vol. 87, pp. 735-742, March 1968.

[7] P. Silvester, "Skin effect in multiple and polyphase conductors," IEEE TRANS. POWER APPARATUS AND SYSTEMS, vol. 88 , no. 3, March 1969.

[8] H. B. Dwight, Electrical Coils and Conductors. New York: McGrawHill, 1945 .

[9] P. E. Burke, R. T. H. Alden, "Current density probes," IEEE TRANS. POWER APPARATUS AND SYSTEMS, vol. 88, pp. 181-185, February 1969.

\section{ACKNOWLEDGEMENT}

The authors wish to acknowledge financial support of this research by the National Research Council of Canada.

\section{Discussion}

A. A. Halacsy (University of Nevada, Reno, Nev. 89507): The authors calculate the current-distribution in the cross-section of the conductor as the function of the frequency. The question arises, what happens at d.c., that is at zero-frequency? It is true that no skin-effect exists at d.c. but it is also true that unidirectional currents pull together as can be seen, for instance, at any electrometalurigical plant having several parallel and not insulated cables carrying several ten thousands of amperes.

Figure 8 shows a reduction of the number of subdivisions in the inner part of the conductor. This can be done only in the inner part or rather, in a part of the cross-section where the current density is smaller than in other parts. This procedure requires, therefore, a knowledge of the very current-distribution which is to be calculated.

Manuscript received August 2, 1971.

J. H. McWhirter (Westinghouse Electric Corporation, Pittsburgh, $\mathrm{Pa}$ 15235): This paper is one more in a succession of fine papers on the numerical solution of electromagnetic field problems which have come from Prof. Silvester and his associates.

Manuscript received August 6, 1971
Problems of this type may be formulated using either the concept of a field or the concept of equivalent circuit. The field concept is the more powerful approach although the other may provide more insight as a carryover from circuit theory. In both cases, either "direct" or modal solutions may be used and there is no need to associate one concept of problem formulation with a particular solution.

I have used "direct" methods for the solution of matrix equations which approximate eddy current field problems. While the matrices do tend to be ill-conditioned, the results obtained are believed to be reliable and some care has been exercised to verify this. Are the authors referring to ill-conditioning of matrices when they speak of instabilities? Are these instabilities such as to be overcome by the use of higher precision arithmetic and/or iterative methods in the solution?

I recognize the advantage of the modal method in that the computational economies are better and that much of the solution can be performed independent of frequency. The frequency independence will not be a consideration in a large class of problems but, of course, will be a factor in other problems.

Is the modal method equally advantageous in the solution of say, antenna problems, as compared to direct methods which are commonly used?

The computational advantage of the modal method over the direct method appears to exceed the complications of the eddy current problems over those of static problems (Poisson's equation). This suggests the thought that modal techniques may have some usefulness in the solution of static field problems as formulated from integral equations. I do not know how this would be done and it may be a foolish thought but I would be interested in the author's reaction.

I would tend to favor definite approximation functions for solutions to problems of this type and the use of either exact analytical integrations or numerical integrations in order to obtain the matrix coefficients. For example, in this problem, triangular elements instead of rectangular elements could be utilized and the current density could be assumed to be linear over each triangle and continuous between triangles. Do the authors see any advantages or disadvantages to this viewpoint?

P. Silvester, S. K. Wong and P. E. Burke: The authors wish to thank the discussers for their evidently active interest in this paper.

While the method described in this paper calculates in the first instance only the distribution of alternating currents in the coils under analysis, electromagnetic forces between the coil turns, or between the coil turns and any other object, can be found once the current distribution is known. This applies both to alternating current carrying coils, and to the direct current case that Dr. Halacsy alludes to.

Dr. Halacsy, of course, is perfectly correct in observing that the variable-sized subdivision used in the paper presupposes some prior knowledge about current distribution in the coil turns. It should be noted, however, that this prior knowledge is exploited to lower computing costs; it is not a necessary requirement of the method. For example, if one had no knowledge whatever of the current distribution to be expected, one might subdivide the turn into equal-sized parts, calculate the current distribution, and then re-adjust the subdivision so as to achieve the highest precision wherever the current density appears to vary most rapidly. In this way, two small problems can be solved instead of one large one, thereby saving considerable computing cost. Mr. McWhirter is correct in assuming that our reference to numerical instabilities encountered in "direct" methods alludes to matrix illconditioning. The authors' experience has been variable in this matter, some problems showing poor conditioning, while others, not obviously different physically, have produced stable and useful answers. There is no doubt that iterative clean-up could improve those with relative instability to a very great extent. For precisely this reason, it is improbable that the modal method will find wide application in the solution of static field problems. In time-varying problems, such as the antenna problem, however, matters are frequently different. There, modal analyses have been found useful in the past by various workers; and there is little doubt that their usefulness will grow in time.

The choice of rectangular regions, as used in this paper, is of course arbitrary; triangles or other shapes could be used instead. There do arise certain difficulities in two-dimensional numerical integration over regions of comparatively irregular shape, which are avoided by the use of rectangles. On the other hand, triangles or arbitrary convex quadrilaterals have obvious advantages in their ability to model very complicated shapes with high accuracy.

Manuscript received September 1, 1971. 